\title{
Dorsal and Ventral Myotome Motoneurons and Their Input During Fictive Locomotion in Lamprey ${ }^{1}$
}

\author{
P. WALLÉN, S. GRILLNER, ${ }^{2}$ J. L. FELDMAN, ${ }^{3}$ AND S. BERGELT \\ Department of Physiology III, Karolinska Institutet, Lidingövägen 1, S-114 33 Stockholm, Sweden
}

\begin{abstract}
Motoneurons supplying the dorsal and ventral parts of the myotome in the lamprey are shown to have different morphological characteristics; furthermore, their pattern of activation during fictive locomotion may differ considerably. Intracellular recordings from motoneurons were performed in an in vitro spinal cord-myotome preparation from segments rostral to the fins. The location of the contracting muscle fibers in the myotome could be observed directly in the dissection microscope during intracellular stimulation of the motoneuron. The motoneurons were injected with Lucifer Yellow, an intracellular dye, and were subsequently reconstructed, sometimes in both a horizontal and a transverse plane. Motoneurons supplying the ventral third of the myotome had a dense, fan-like, dendritic tree and ramifications near the midline. In contrast, motoneurons supplying the dorsal third of the myotome had a more widespread and less dense dendritic tree, with few ramifications near the midline. Some motoneurons supplying the most ventral or dorsal part of the myotome had contralateral dendrites crossing in the ventral commissure and ramifying near contralateral large, reticulospinal Müller fibers. The differences in morphology may indicate that these motoneurons receive different descending inputs. This may be related to the need for an effective control in the dorsoventral plane during righting and steering responses. During fictive locomotion elicited in the isolated spinal cord by bath-applied $\boldsymbol{N}$-methyl-aspartate, pairs of motoneurons were recorded which subsequently were identified and characterized by intracellular injections of Lucifer Yellow. Motoneurons with similar morphology had similar drive signals, i.e., membrane potential oscillations during fictive locomotion. In contrast, motoneurons with a morphology suggesting that they supply different parts of the myotome could have a very different input signal with even differing phase relations. The consequences for the segmental pattern-generating circuitry are discussed.
\end{abstract}

Received May 4, 1984; Revised July 26, 1984;

Accepted August 28, 1984

\footnotetext{
${ }^{1}$ We would like to thank Dr. T. L. Williams for valuable comments on the manuscript. The skillful help with typing (I. Klingebrant), histology ( $H$. Axegren), and technical matters (G. Goertz and W. Johansen) is also gratefully acknowledged. This study has been supported by the Swedish Medical Research Council (Project No. 3026, and Visiting Scientist Fellowship to J. L. F., Project No. 6224) and by Karolinska Institutets fonder and M. Bergvalls stifteise. J. L. F. was supported in part by Research Career Development Award HL-00554 from the United States Public Health Service.

${ }^{2}$ To whom correspondence should be addressed.

${ }^{3}$ Present address: Departments of Physiology and Anesthesia, Northwestern University, Chicago, IL 60611.
}

The lamprey nervous system in vitro can be used to study the neuronal mechanisms underlying motor coordination and, in particular, locomotion. The swimming motor pattern is elicited by alternating contraction of the motor units on the opposite sides of one segment and a delayed activation of more caudal segments, which results in an undulatory wave traveling down the body with increasing amplitude.

This study is concerned with the morphology of the different motoneurons supplying the body wall in one segment and how they are activated during fictive locomotion. Somatic motoneurons have already been demonstrated to receive periods of alternating excitation and inhibition (Russell and Wallén, 1980, 1983; Buchanan and Cohen, 1982; Kahn, 1982). Motoneurons of one somatic segment have been assumed to constitute a functionally homogeneous population divided only into motoneurons supplying fast and slow muscle fibers. The latter type tends to be somewhat smaller and tends to have a higher input resistance (Teräväinen and Rovainen, 1971). Motoneurons are described as being located in a longitudinal cell column without clear segmentation and having a flattened, fanlike dendritic tree oriented in a transverse plane on the ipsilateral side (Tretjakoff, 1909; Teräväinen and Rovainen, 1971; Tang and Selzer, 1979).

In the present study we report that the detailed morphology of motoneurons supplying the body wall is much more varied than was previously assumed. Motoneurons supplying the dorsal part of one segment differ from those supplying the most ventral aspect. The input to these two categories may be quite different during fictive locomotion.

\section{Materials and Methods}

The spinal cord of lampreys (/chthyomyzon unicuspis or, in two cases Petromyzon marinus) was dissected in two different ways. (1) An isolated spinal cord preparation was dissected free from the notochord, and great caution was taken to cut ventral roots at some length, so that they could be used for recording with suction electrodes. The spinal cord was pinned down with the ventral side up in a Sylgard-lined dish, after the meninx primitiva had been removed on the dorsal and the ventral sides. Care was taken to fix the spinal cord at the same length it had in situ. Under these conditions cell contours could easily be seen in the dissection microscope, when the spinal cord was transilluminated. This condition facilitated successful microelec trode penetration of probable motoneurons. (2) The second preparation was a modified spinal cord-myotome preparation (cf. Teräväinen and Rovainen, 1971). Spinal cord segments located from just caudal to the gill region but rostral to the dorsal fin and anus were used, i.e., a region containing only muscles of the body wall but not any fin musculature. A dorsal midline incision was made and the muscles on one side were removed (see Fig. $2 C$ ). Subsequently, the spinal canal was opened and the notochord was cleared ventrally. The remaining body segments were skinned. Care was taken not to damage muscle fibers even on the most dorsal or ventral aspect of the hemisegments. The notochord was pinned down in a Sylgard-lined chamber in which a trough provided sufficient space for the ventral part of the body wall. The dorsal side of the spinal cord was stripped of the meninx primitiva. The preparation was photographed in Polaroid at the onset of the experiment so that the location of both impaled motoneurons and the muscle contraction elicited by intracellular stimulation could be accurately mapped. 


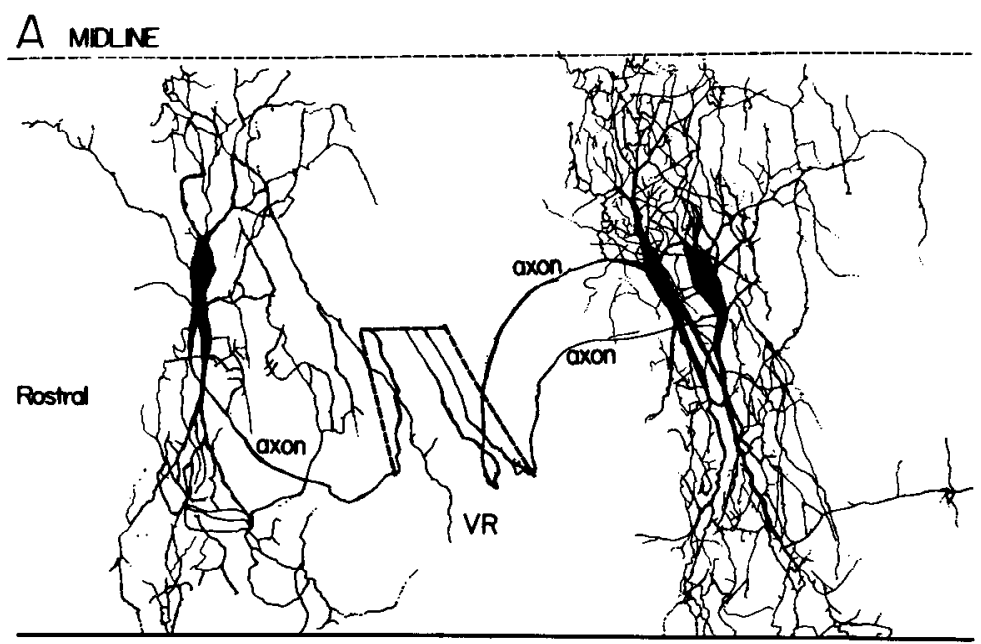

$\mathrm{B}$

LATERAL MARGN
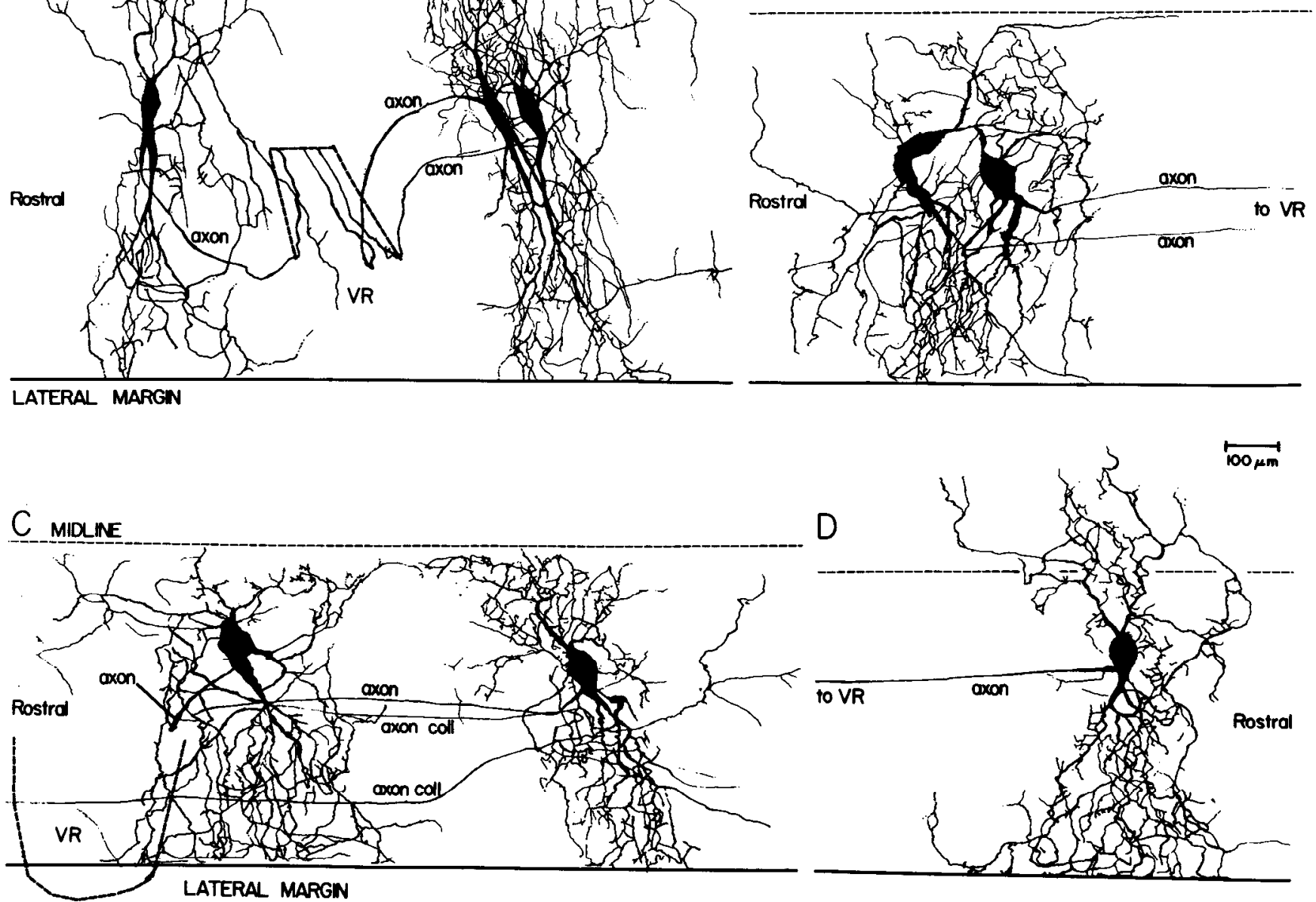

Figure 1. Reconstructions of motoneurons recorded in the isolated spinal cord preparation. $A$, Three motoneurons with their axons in the same ventral root (VR); which is indicated with heavy dashed lines (ventral cord surface is upward). In this and subsequent illustrations the lateral margin of the spinal cord is marked with a solid line and the midline is marked with a dashed line. $B$. Two closely spaced motoneurons with their axons running in the caudal direction. C, Both motoneurons have axons in a ventral root located rostrally. The caudal cell has, in addition, two axon collaterals (axon coll). $D$, Another motoneuron with its axon pointing caudally and with extensive contralateral dendrites.

The frequency of tetanic fusion of the contraction was determined visually through the dissection microscope, as previously described (Rovainen and Birnberger, 1971; Rovainen, 1974).

Neurons were impaled with Lucifer Yellow-filled electrodes $(5 \%$ in $0.1 \mathrm{~mm}$ $\mathrm{LiCl}$; Stewart, 1978), and the dye was injected by hyperpolarizing current (1 to $2 \mathrm{nA}$ for 20 to $30 \mathrm{~min}$ ). Cells were identified as motoneurons $(n=97)$; (1) if an action potential could be recorded in an adjacent ventral root on intracellular stimulation (Russell and Wallén, 1983) and/or (2) if the axon of the dye-injected cell could be traced into the ventral root.

In the isolated spinal cord preparation, the motor pattern underlying swimming was elicited by adding an excitatory amino acid, $N$-methyl-aspartate (50 to $150 \mu \mathrm{M}$ ) to the bath (Cohen and Wallén, 1980; Poon, 1980; Grillner et al., 1981). The efferent motor pattern was recorded in ventral roots by suction electrodes and by two microelectrodes located in different motoneurons, which were between $25 \mu \mathrm{m}$ and one segment apart.

The recording chamber was kept at 6 to $8^{\circ} \mathrm{C}$ and contained physiological solution (Wickelgren, 1977).

All spinal cords were fixed in formaldehyde $(4 \%)$ in buffer for $1 \mathrm{hr}$, dehydrated in ethanol in steps from $70 \%$ to $99 \%$, and cleared in methylsalicylate (see Stewart, 1978). The spinal cords were mounted as whole mounts and photographed (Tri-X film pushed to 1600 ASA) in 20 to 40 focal planes from dorsal to ventral in a UV-fluorescence microscope (Leitz Diavert). Reconstructions were made from the negatives projected by an enlarger. In our hands the reconstructions appeared more accurate when performed in this way rather than with a drawing tube. Some preparations were chosen for transverse sections (see Fig. 2) to allow a more detailed analysis of the relations between cell processes and axons. They were then embedded in paraffin, sectioned in 10- $\mu \mathrm{m}$ sections, and mounted on slides. Subsequently, these were photographed and reconstructions were made as described for the whole mounts.

\section{Results}

\section{General characteristics of motoneurons supplying the body wall}

All of the motoneurons studied ( $n=97$ ) were located in spinal cord sections rostral to the first dorsal fin but caudal to the gills (see Fig. 2C). They controlled some aspect of the myotomes from the most ventral to the most dorsal aspect. Below we will describe the general features of the different parts of the motoneuron.

Axon. The axon originates from a cornet-like axon hillock (Figs. 1 to 5) located on the cell body or occasionally on a proximal dendrite on the lateral or the medial side. The axon hillock points in a ventral and either a rostral or caudal direction. The axon gradually shifts to a position in the ventral tract of axons (Fig. 2, B, E, and $H$ ), and it continues in either the rostral or the caudal direction (Figs. 1 and 5 ) to bend; sometimes very sharply, and to exit into the ventral root. The ventral roots are flattened and the axons are arranged in an orderly fashion so that the most caudal axon belongs to the caudalmost motoneuron. The motoneurons appear in a continuous column without segmental subdivision (Fig. 5), and of the motoneurons located in the region between two ventral roots, those in the rostral 

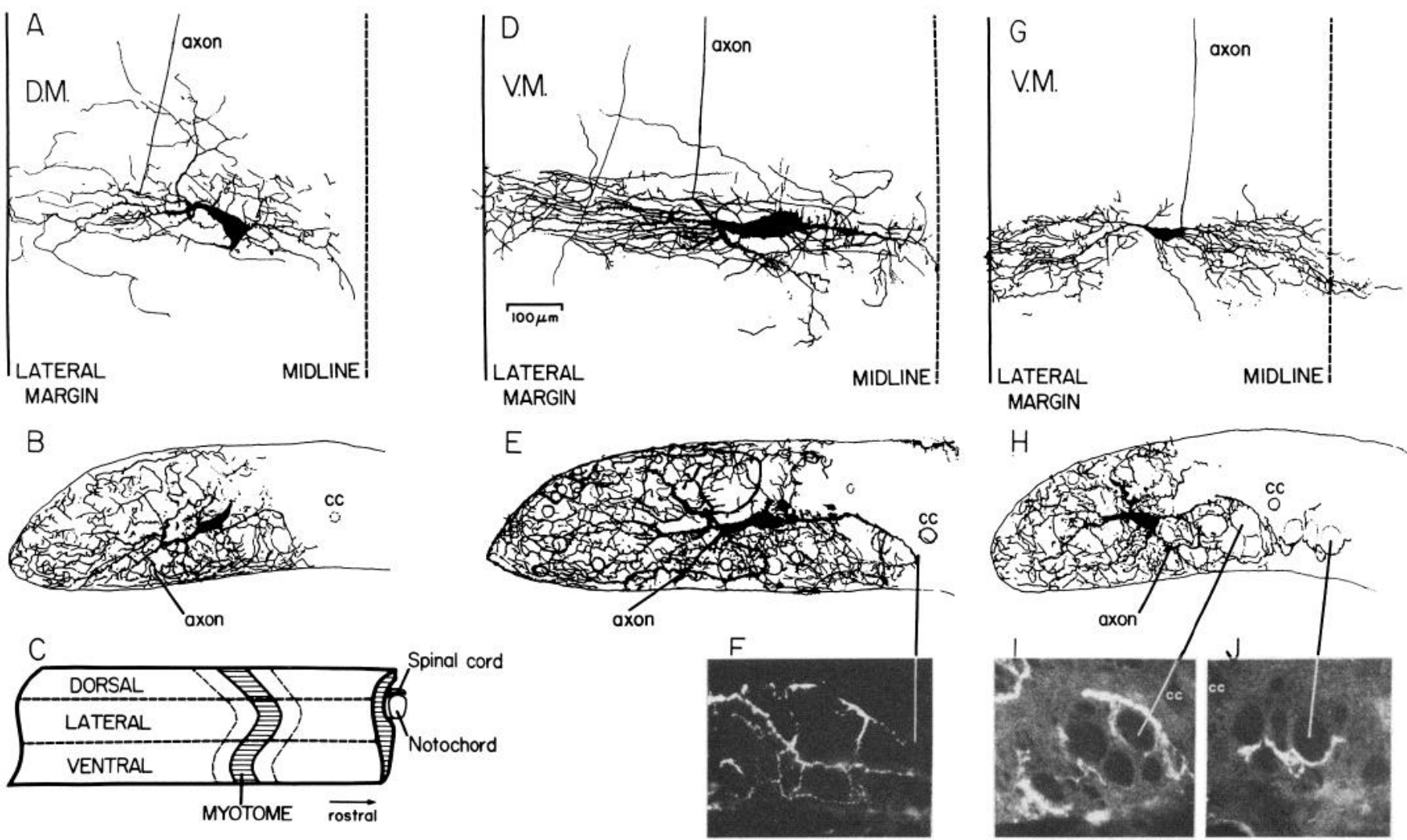

Figure 2. Morphology of motoneurons supplying different parts of the body wall. $A$ and $B$ are whole mount and transverse reconstructions, respectively, of a dorsal myotome (D.M.) motoneuron. The location of the axon and the central canal $(C C)$ is indicated. The drawing in $C$ depicts the preparation used, with one-half of the musculature left intact. One of the segmental myotomes is indicated as well as the subdivision of the body wall into three parts of approximately equal surface area. $D$ and $E$ illustrate, in the same way as $A$ and $B$, a ventral myotome (V.M.) motoneuron. $F$ is a photomicrograph of the fluorescent dendritic branches in the medial region, passing around the large Müller fibers. $G$ and $H$ show a ventral myotome (V.M.) motoneuron with contralateral dendrites. I and $J$ are micrographs from the ipsilateral and contralateral medial regions, respectively. The dye-filled branches appear as white strands close to the dark Müller fibers. cc, central canal. The tetanic fusion frequency was determined for the neuron in $\mathrm{G}$ to $\mathrm{J}$ as $6.5 \mathrm{~Hz}$.

half of the region exit in the rostral root, whereas those in the caudal half exit in the caudal root.

All axons have a characteristic thin initial segment, but the axon diameter subsequently increases. Some axons increase further in caliber as they enter into the ventral root (Fig. 3C, see "Discussion"). As a rule the axon progresses along a straight course without further subdivisions, but, occasionally, as in the right cell of Figure $1 C$, axon collaterals may occur. In this case two branches probably exit through the same ventral root, while the third branch clearly passes lateral to the origin of the ventral root to continue to an unknown destination. In most cases the axon hillock has one or several long processes which morphologically cannot be distinguished from dendrites originating from the cell body.

Soma. The shape and the size of the soma are quite variable (Figs. 1 to 5 ) and presumably are influenced by the points of origin of the larger dendrites. The soma is often disc-shaped and tapers out into the medial and the lateral dendrites (Fig. 1). A round or multipolar shape is also common (Figs. 1 to 5 ).

Dendrites. The dendrites extend as a rule from the lateral margin to near the midline and in some cases even to the contralateral side as far as halfway between the midline and the lateral margin (Figs. 1 to 5 ).

From the reconstructions in the transverse plane (Fig. 2, B, E, and $H$ ), it is evident that medial dendrites are juxtaposed to some of the large medial Müller axons (reticulospinal) on the ipsilateral and sometimes also on the contralateral side (see also below). On the ipsilateral side the dendrites ramify preferably in the ventral and lateral aspect and can thus be seen to be in close contact with many axons. The dorsal column is always spared and ramifications in the region just lateral to the dorsal column are also scarce. In addition to some large dendritic branches, there are often a variety of smaller dendrites originating from the soma. The thinnest dendrites may be very short and without branches (Fig. 1).

In some cells the rostrocaudal extent of the dendritic tree is small and often very dense, and it can, then, rightly be described as fanlike (Figs. $1 A$ and 2, $D$ and $G$ ). In many instances, however, the rostrocaudal extent is nearly as large as the mediolateral (Figs. $1 C$, $2 A$, and 5 ). Such a form has previously been thought to be specific for fin motoneurons (Rovainen and Birnberger, 1971).

\section{Subdivision of motoneurons based on which part of the body wall they supply}

The muscle fibers in the most dorsal aspect of a segment, near the midline, will obviously exert different mechanical effects from ventrally located muscle fibers, which in turn will differ from fibers just lateral to the notochord (see Fig. $2 \mathrm{C}$ ). Although all will contribute to a lesser or greater extent to a lateral bending movement, ventral and dorsal muscle fibers will be antagonists in movements in a dorsoventral direction.

To test whether the varied morphology of motoneurons discussed above was related to which part of the body wall they supply, a spinal cord-myotome preparation was used, in which all muscle fibers on one side of a section of the body were left intact (cf. "Materials and Methods" and Teräväinen and Rovainen, 1971). After impaling a motoneuron, it was stimulated by current pulses. The resulting muscle fiber contraction could easily be seen through the dissection microscope and the exact location on the body wall could be determined.

Figure $2, D$ to $F$, shows a motoneuron which supplies a muscle 
A

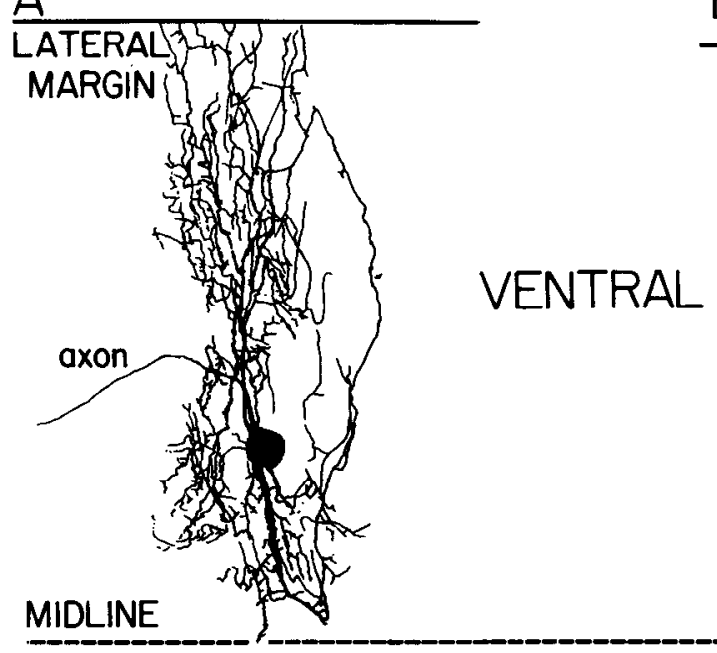

B

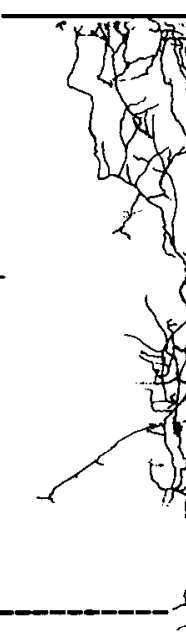

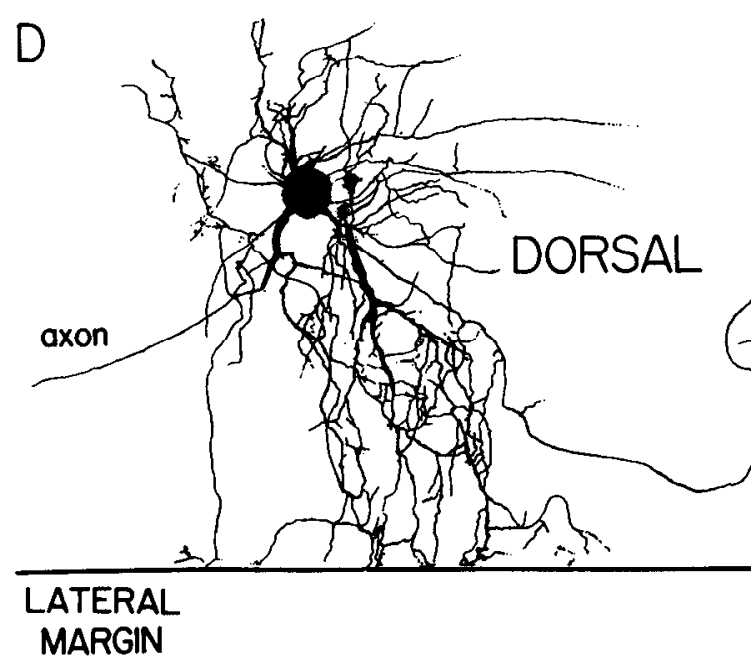
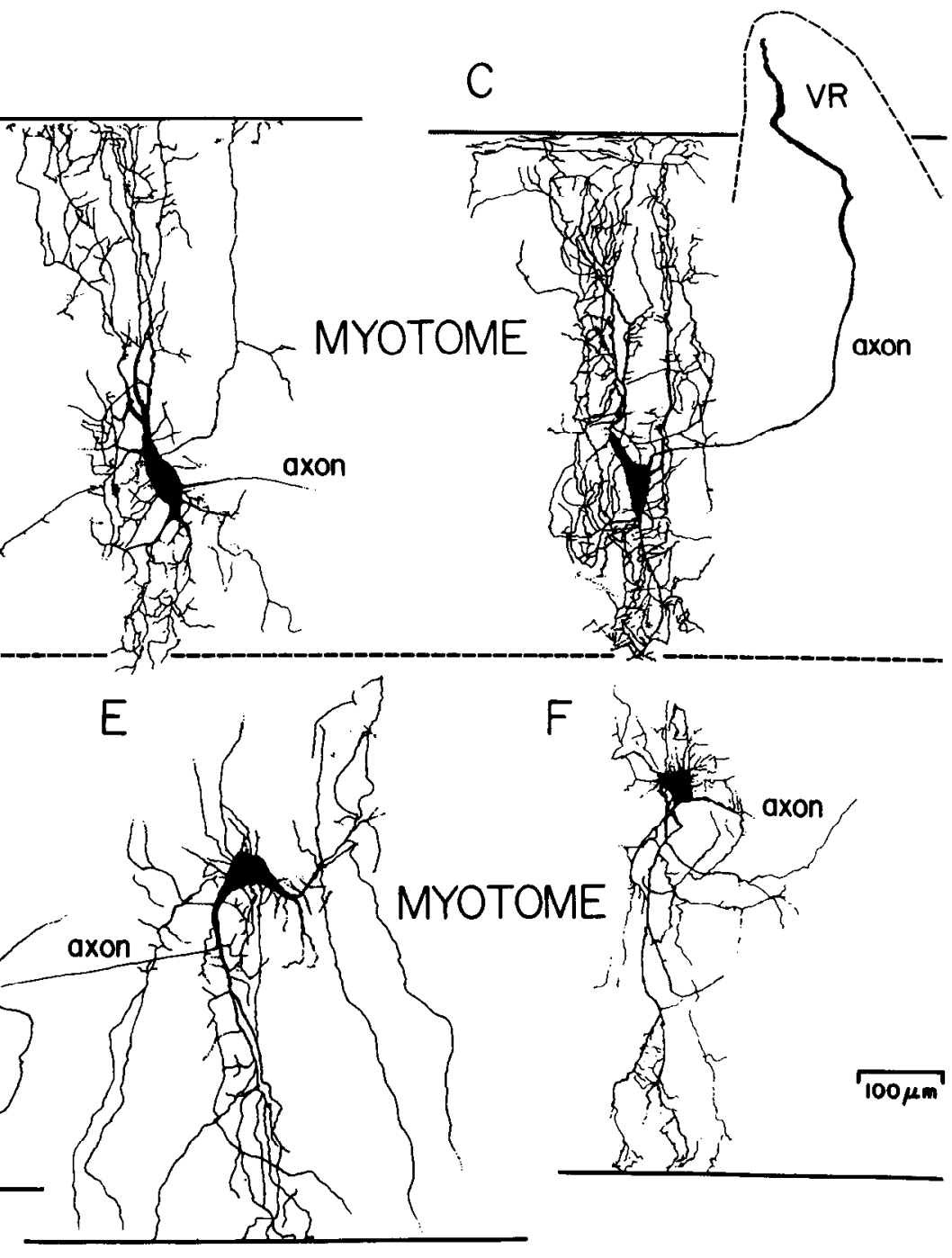

Figure 3. Different morphology of ventral and dorsal myotome motoneurons. $A$ to $C$ are motoneurons supplying the ventral aspect of the myotome; $D$ to $F$ are dorsal myotome motoneurons. The midline (heavy dashed line) is common to all six cells. Fusion frequencies for tested neurons were: $A, 7.7 \mathrm{~Hz} ; B$, $10.5 \mathrm{~Hz} ; C, 8.7 \mathrm{~Hz} ; D, 6.7 \mathrm{~Hz} ; E, 6.3 \mathrm{~Hz}$.

unit in the ventral third of the myotome (see Fig. $2 C$ ). From the horizontal and transverse reconstructions it is apparent that the medial dendrites extend all the way to the midline and ramify around the most medial Müller fibers (which originate in the mesencephalon; Rovainen, 1974) and in the ventromedial area (Fig. 2, $E$ and $F$ ). In addition, there are rather dense ramifications in the dorsolateral aspect. Figure $3, A$ to $C$, shows the morphology of three additional ventral myotome motoneurons. Note the extensive medial ramifications near the midline, the tendency for a mediolateral orientation of dendrites, and an elongated shape of the cell bodies.

Figures $2, A$ and $B$, and $3, D$ to $F$, show motoneurons which supply the dorsal part of the myotome. In this category the medial dendrites tend to be more widely distributed in the rostrocaudal extent and they do not ramify as densely in the medial area around the two or three most medial Müller fibers. Numerous ramifications occur in a somewhat more lateral region which includes the more lateral bulbospinal Müller axons and the ventral margin. Their cell bodies tend to have a multipolar or pear-shaped form (Figs. $2 A$ and $3, D$ to $F$ ).

A limited number (total 17) of motoneurons have been found to have contralateral dendrites (Figs. 2, G to $J$, and 4). In the motoneuron of Figure 2, $G$ to $\mathrm{J}$, the dendrites cross in a ventral location below the central canal to ramify near the contralateral medial Müller axons (see Fig. 2J). Figure 4 shows two additional examples of motoneurons with contralateral dendrites, one supplying the ventral part of the myotome (Fig. 4, V.M.) and the other supplying the dorsal aspect (Fig. 4, D.M.). Motoneurons with crossed dendrites supply only the most dorsal or ventral aspect of the body wall.

The motor units have been divided into dorsal (Figs. 2, $A$ and $B$, and $3, D$ to $F$ ), lateral, and ventral (Figs. $2, D$ to $J$, and $3, A$ to $C$ ) groups as shown in the inset of Figure $2 C$. Motor units of the lateral group have an intermediate morphology and have not been illustrated here. In contrast to fish, the myotome of the lamprey is not divided into a clearly separable dorsal epaxial part and a hypaxial part (Teräväinen, 1971). In the lamprey there are no clear dividing lines. We will therefore utilize this arbitrary tripartite subdivision and, below, we will refer to motor units as ventral myotome, lateral myotome, or dorsal myotome motoneurons (Fig. $2 \mathrm{C}$ ). Of a total of 20 ventral and 17 dorsal myotome motoneurons, there was never any overlap with regard to the differences discussed above; thus, they clearly form two distinctly separable groups.

\section{Simultaneous recordings of pairs of motoneurons}

In the isolated spinal cord, the motor pattern underlying locomotion was elicited by adding $N$-methyl-aspartate to the bath (see "Materials and Methods"). Pairs $(N=16)$ of motoneurons were recorded during 


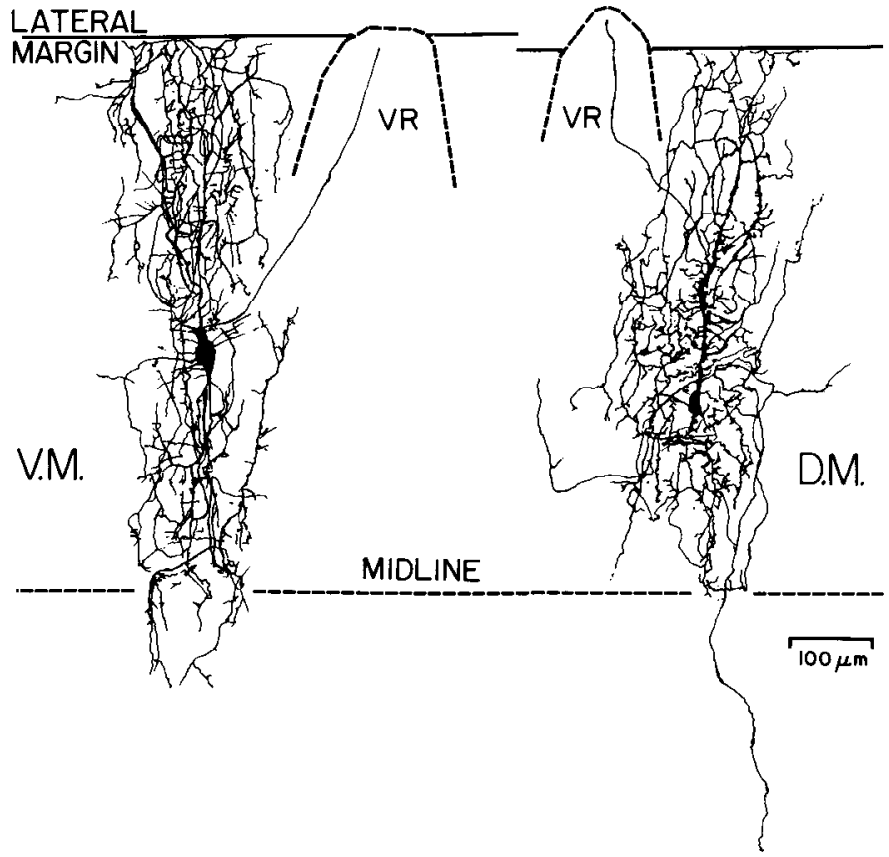

Figure 4. Motoneurons with contralateral dendrites. The two cells supplied the most ventral (V.M.) and the most dorsal (D.M.) aspects of the body wall, respectively. Note the density of ramifications near the midline in the V.M. cell and the relative scarcity in this region in the D.M. cell. Fusion frequencies were: V.M. cell, $8.9 \mathrm{~Hz} ; D . M$. cell, $5.9 \mathrm{~Hz}$.

such fictive locomotion, and the oscillations were correlated to the overall efferent activity sampled from the ventral roots. Simultaneous recordings from two motoneurons disclosed that the membrane potential variations can be very similar, as in Figure $5 B$, although one is spiking and the other one is not. These two motoneurons are located very close to each other, but they send their axons to separate ventral roots and hence represent separate segments. It is often assumed that interneurons are subdivided so that there is a separate control over each segment. In that case the two motoneurons would have been expected to have a somewhat different input. It should be noted that the two motoneurons have similar morphological characteristics. Figure $6 A$ is from another pair of motoneurons (Fig. $1 A$ ) with similar characteristics and similar input, although they are at some distance from each other within one segment.

The pair of motoneurons of Figure $5 A$ are at some distance from each other but belong to the same segment, as their axons exit through the same ventral root. In this case the membrane potential oscillations differ markedly. The lowermost recording is from the cell to the left; the depolarizing phase may start about one-fourth of a cycle before that of the other motoneuron, and the same applies to the hyperpolarizing phase. These two motoneurons are very different from a morphological point of view. The left and right neurons resemble those supplying the ventral and the dorsal musculature, respectively. Figure $6 B$ is from a series of records from two motoneurons which differ markedly but not entirely in the type of oscillation. They are from the pair in Figure $1 B$, in which the neurons are morphologically dissimilar but located very close to each other. We encountered 4 pairs of this type and 12 pairs of the type discussed above in which the oscillations followed each other closely and the morphology was similar

In some rhythmically active neuronal systems, the extracellular field potential has been found to vary by several millivolts in phase with the rhythmic activity (Jefferys and Haas, 1982). Under the present conditions such variations are negligible (Fig. $6 \mathrm{C}$ ). The field potential recorded inside one spinal neuron or extracellularly when a nearby neuron is spiking may nevertheless exceed $1 \mathrm{mV}(P$. Wallén, J. L. Feldman, and S. Grillner, unpublished observation).
The field potentials caused by activity in different nearby neurons appear to cancel each other since they are desynchronized in time.

\section{Discussion}

Considerations related to the morphology of motoneurons. The myotome-spinal cord preparation used in the elegant experiments of Rovainen, in both the motoneuron study (Teräväinen and Rovainen, 1971) and the study of Müller cell projections to motoneurons (Rovainen, 1974), lacked the dorsal third of the myotome, which was removed to improve the accessibility of the spinal cord. Hence, neither the input of these motoneurons nor their characteristics have been studied before. The availability of better intracellular dyes like Lucifer Yellow (Stewart, 1978), which fill the distal dendrites, has obviously improved the possibilities for making detailed reconstructions of the motoneurons. The motoneurons supplying the ventral third have a more fan-like dendritic tree extending to the midline, whereas motoneurons to dorsal muscles have a more scattered and wide dendritic tree with few ramifications near the midline (Figs. 2 and 3). These two types appear similar to the type I and type ॥ motoneurons described by Tretjakoff (1909). The latter type was not found by Teräväinen and Rovainen (1971) in somatic segments, presumably due to their removal of the dorsal muscles, but motoneurons supplying the dorsal fin were described as type II (Rovainen and Birnberger, 1971). Tretjakoff (1909) described some motoneurons that were identified by tracing the axon into the ventral root (both type I and type II), but, unfortunately, he also named as motoneurons a number of cells which only had a general similarity to motoneurons. Judging from his illustrations, they include a number of cells which now appear unlikely to be true motoneurons.

The ventral myotome motoneurons have extensive ramifications around the medial Müller axons (Fig. 7) and the adjacent ventromedial area in which 5-hydroxytryptamine $(5-H T)$ neurons form a dense plexus (Baumgarten, 1972; van Dongen et al., 1983a, b, c). The dorsal myotome motoneurons have few branches in this area but extensive ramifications around the bulbar Müller axons and the lateral extension of the 5-HT-plexus. In the light microscope it is impossible to ascertain whether synapses are formed or whether the dendrites just extend in the narrow space in between the different large axons in the "white" matter (see, however, Fig. 2, $H$ to $J$ ). Monosynaptic connections to motoneurons have been demonstrated physiologically from both medial and more lateral Müller axons (Rovainen, 1974). If syrlapses are formed to these dendrites, one would predict that this would occur preferentially between medial Müller axons and ventral myotome motoneurons and that some bulbospinal Müller axons would activate the dorsal group. This has to be tested in direct experiments. It should be noted, however, that the medial Müller cells ( $M 1$ to $M 3$ and 11) are inhibited by some vestibular afferents which excite the bulbar group (B1 to B4) and vice versa and, furthermore, that stimulation of one vestibular nerve may cause excitation of the dorsal part of the myotome while other inputs activate the ventral part (Rovainen, 1979, 1983). The bulbar group can also influence the swimming motor pattern (Buchanan and Cohen, 1982).

Motoneurons with crossed dendrites evidently will have input from the contralateral side. The motoneuron shown in Figure 2, $G$ to $J$, supplied the extreme ventral aspect of the body wall, and it is noteworthy that ramifications pass in close proximity to contralateral medial Müller fibers (Fig. 2J). Other motoneurons with crossed dendrites supplied the most dorsal aspect of the myotome (Fig. 4, $D . M$.), but none was found in the intermediate region. In movements along a dorsoventral axis such as will occur in steering during locomotion or in righting responses, dorsal myotome motoneurons on both sides will act as synergists, being antagonistic to motoneurons supplying the ventral musculature. This situation differs from the activity during the side-to-side undulations of locomotion.

The differences in rostrocaudal extent of the dendritic tree between dorsal myotome motoneurons and ventral myotome motoneurons is puzzling. It would seem somewhat unlikely that one group 

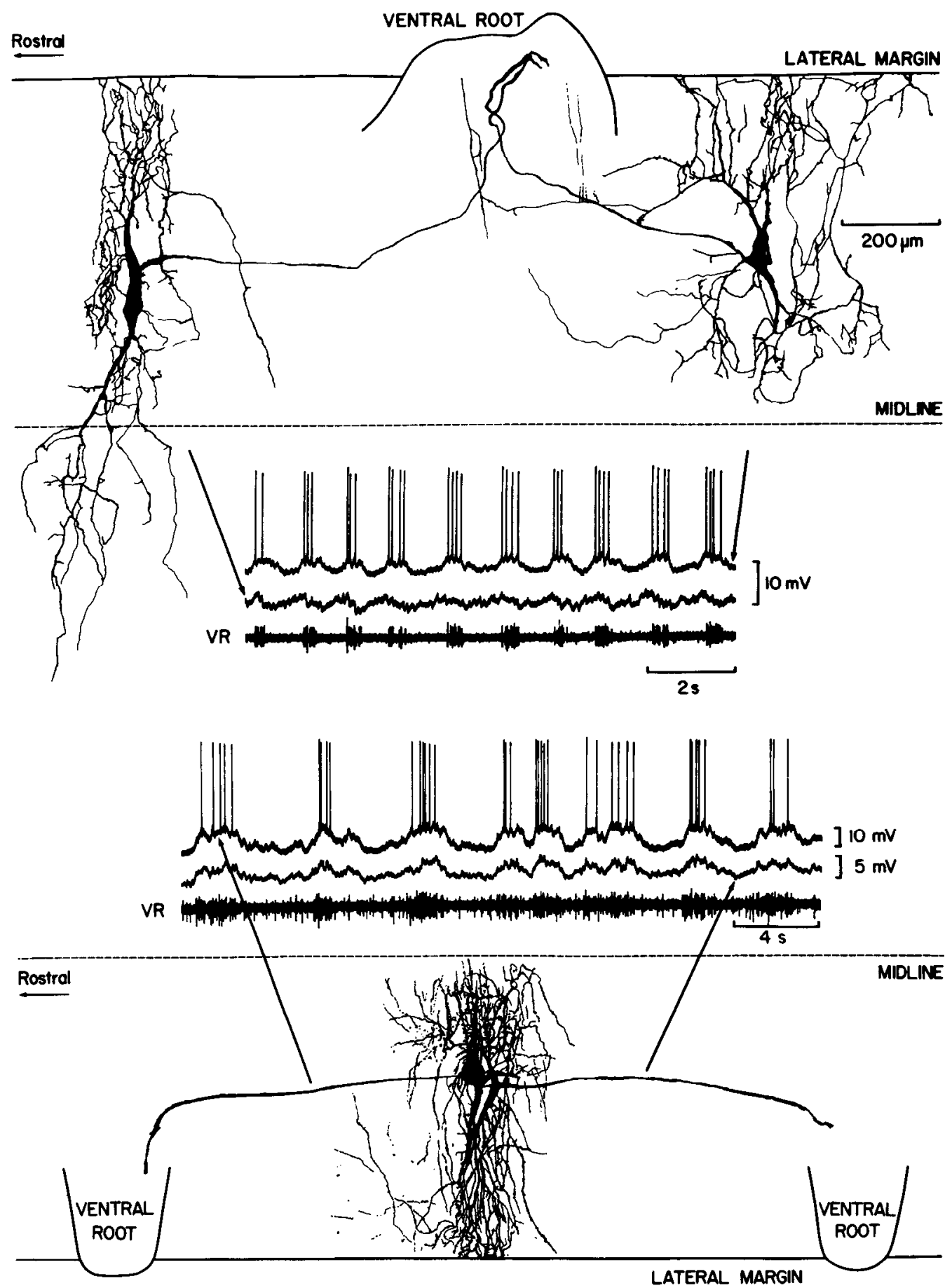

Figure 5. Paired intracellular recordings from motoneurons in the isolated spinal cord preparation. At the top is shown two motoneurons with their axons in the same ventral root (the ventral side of the spinal cord is upward). Their activity pattern during fictive locomotion is shown in the middle as indicated by the arrows, together with the recording from the ventral root (VR). Note the phase difference between the membrane potential oscillations of the two cells and the difference in morphology. The bottom reconstruction illustrates two closely spaced motoneurons with similar morphology. The rostral cell sends its axon to the rostral ventral root and the caudal cell to the caudal root. As seen above, from the recordings during fictive locomotor activity, the rostral neuron (left arrow) fired bursts of action potentials but the caudal cell did not. Nevertheless, the membrane potential oscillations in the two cells were very similar. The ventral root record $(V R)$ is from the caudal root.

should pick up signals from a larger part of a segment than the other. One explanation might be found in the anatomical arrangement of motoneurons within the gray matter. However, no clear mediolateral organization in relation to these types of motor units has become apparent, but we have not analyzed the relative dorsoventral position of cells within the thin gray matter of the lamprey spinal cord. If the dendrites have to circumvent cell bodies to reach the fiber tracts, their course will differ as compared to the case when the proximal dendrites are juxtaposed to the fiber tracts.

Significance of differences in input to motoneurons during fictive locomotion. The ability to generate rhythmic coordinated activity is distributed along the spinal cord since pieces of a few segments (down to 1.5 segments) can generate reciprocally organized rhythmic activity (Cohen and Wallén, 1980; Sigvardt and Grillner, in
Grillner et al., 1982). Moreover, there is a phase shitt so that each caudal segment will be activated about $1 \%$ of the cycle duration after its rostral neighbor (Wallén and Williams, 1984), which amounts to a significant delay over the 75 to 100 segments normally present in a cyclostome or a fish swimming forward. Although there is no apparent segmental organization of the gray matter (Tretjakoff, 1909; Rovainen, 1983), the recurrent dorsal and ventral roots certainly subdivide the spinal cord into segments. It has therefore been common to assume as a first approximation that the spinal cord has a series of segmental pairs of oscillators which normally are phase coupled. The phase coupling can be reversed as in backward swimming by an interaction with the system coordinating the oscillators (see Grillner et al., 1983). In each segment the alternating activity should result from a reciprocal inhibitory interaction between 


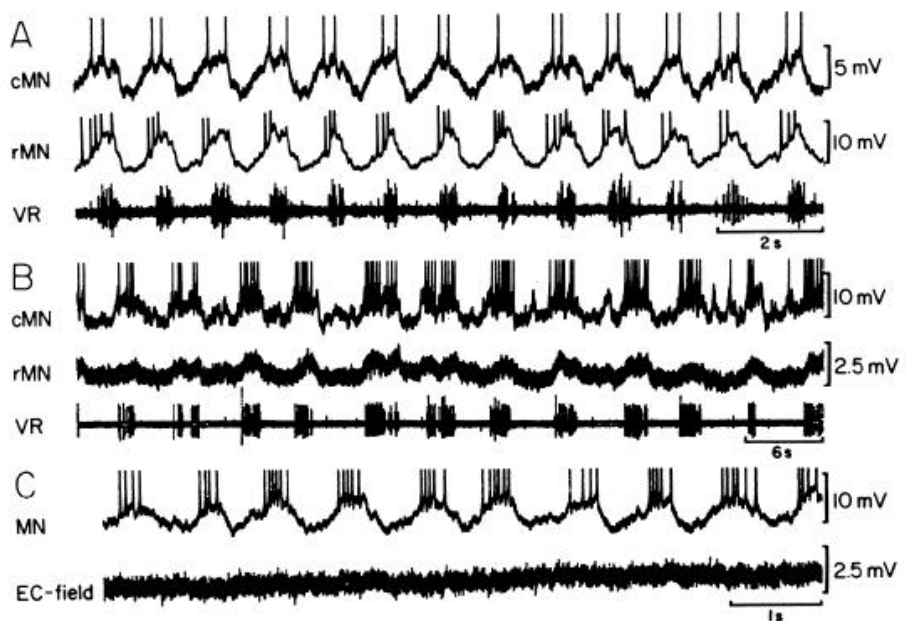

Figure 6. Comparison of the activity in pairs of motoneurons during fictive swimming. $A$, Recordings from the motoneurons illustrated in Figure $1 A$ $C M N$ is from one of the caudal two cells; and $r M N$ is from the rostral motoneuron. Note that the membrane potential oscillations are similar, although the cells were located at some distance from each other. $B$, Records from the motoneuron $(M N)$ pair in Figure $1 B$, which were closely spaced $(C$ caudal; $r$, rostral). Despite this fact, membrane potential oscillations in these cells differed. $C$, Intracellular recording from a motoneuron during fictive locomotor activity, together with the extracellular field recorded with a second microelectrode placed at a distance of about $0.3 \mathrm{~mm}$ from the recorded motoneuron. In $A$ to $C$, action potentials in the top traces have been truncated.

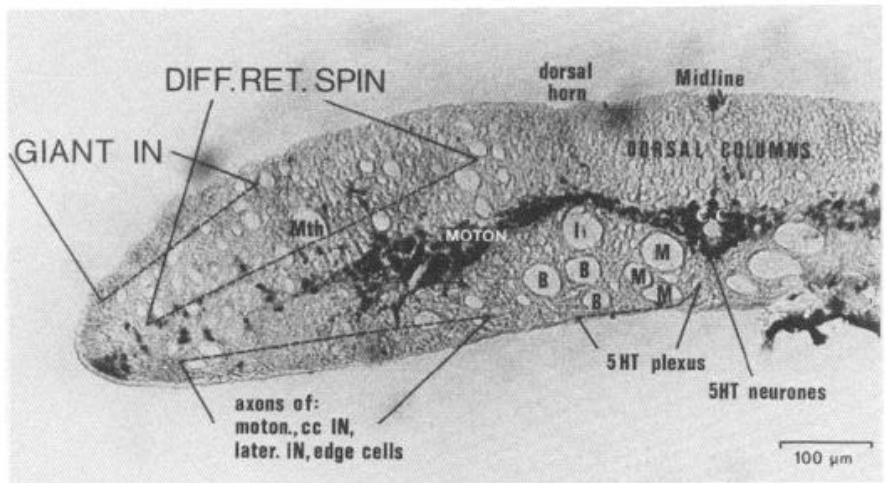

Figure 7. The lamprey spinal cord in transverse section. The regions where axons of different neurons are located are indicated by sectors (solid and dashed lines). Individual Mauthner and Müller cell axons are also indicated. DIFF.RET. SPIN, different reticulospinal axons; GIANT IN, giant interneuron axons; moton., motoneurons; $c c / N$, central canal interneurons: later IN, lateral interneurons. Mth, mauthner cell axon; $l_{1}$, axon of the isthmic Müller cell $I_{1} ; B$, axons of bulbar Müller cells; $M$, axons of mesencephalic Müller cells; CC, central canal. Based on information given in Rovainen (1983) and as referred to in the text.

the oscillators of the two sides. The motoneurons themselves are not part of the pattern-generating system, but their activity represents merely an output from interneurons (Wallén and Lansner, 1983, 1984).

The present results show that some motoneurons within a segment, and even across segmental borders, have similar membrane potential oscillations (Fig. $5 B$ ) but that other pairs of motoneurons have differences not only in amplitudes but also in the phase relations (Fig. $5 A$ ) despite the fact that they supply the same myotome and may be located close to each other (Fig. 6B).

Motoneurons with similar input signals have a general similarity in morphology, but the other pairs appear dissimilar. These types seem to coincide with the dorsal and ventral myotome motoneurons described above. Each segment thus appears capable of a differ- ential activation of motoneurons supplying different parts of the myotome even during fictive locomotion generated by the isolated spinal cord. It is important that this applies also to the phase relation between signals and not only to the relative amplitudes. These results show that the pattern of activation can be more similar across segments than within a segment, which may mean that we have to assume a further subdivision of the circuitry generating the drive signal to motoneurons within each segment, perhaps as a modular organization.

These findings may relate to the need to control the trunk movements in a dorsoventral direction, even during lateral undulations, as during steering and righting responses. In this context it is noteworthy that the motoneurons supplying the dorsal fin in more caudal segments have been reported to be active in antiphase (Buchanan and Cohen, 1982).

\section{References}

Baumgarten, H. G. (1972) Biogenic monoamines in the cyclostome and lower vertebrate brain. Prog. Histochem. Cytochem. 4: 1-90.

Buchanan, J. T., and A. H. Cohen (1982) Activities of identified interneurons, motoneurons, and muscle fibers suring fictive swimming in the lamprey and effects of reticulospinal and dorsal cell stimulation. J. Neurophysiol. 47: $948-960$.

Cohen, A. H., and P. Wallén (1980) The neuronal correlate of locomotion in fish. "Fictive swimming" induced in an in vitro preparation of the lamprey spinal cord. Exp. Brain Res. 41: 11-18.

Grillner, S., A. McClellan, K. Sigvardt, P. Wallén, and M. Wilén (1981) Activation of NMDA-receptors elicits "fictive locomotion" in lamprey spinal cord in vitro. Acta Physiol. Scand. 113: 549-551.

Grillner, S., A. McClellan, K. Sigvardt, P. Wallén, and T. Williams (1982) On the neural generation of "fictive locomotion" in a lower vertebrate nervous system, in vitro. In Brain Stem Control of Spinal Mechanisms, B. Sjölund, and A. Björklund, eds., Fernström Foundation, Series No. 1, pp. 273-295, Elsevier Biomedical Press, New York.

Grillner, S., P. Wallén, A. McClellan, K. Sigvardt, T. Williams, and J. Feldman (1983) The neural generation of locomotion in the lamprey: An incomplete account. In Neural Origin of Rhythmic Movements, A. Roberts, and B. L. Roberts, eds., pp. 285-303, Society for Experimental Biology, Cambridge University Press, Cambridge, England.

Jefferys, J. G. R., and H. L. Haas (1982) Synchronized bursting of Ca1 hippocampal pyramidal cells in the absence of synaptic transmission. Nature 300: 448-450.

Kahn, J. A. (1982) Patterns of synaptic inhibition in motoneurons and interneurons during fictive swimming in the lamprey, as revealed by $\mathrm{Cl}^{-}$ injections. J. Comp. Physiol. 147: 189-194.

Poon, M. L. T. (1980) Induction of swimming in lamprey by L-DOPA and amino acids. J. Comp. Physiol. 136: 337-344.

Rovainen, C. M. (1974) Synaptic interactions of reticulospinal neurons and nerve cells in the spinal cord of the sea lamprey. J. Comp. Neurol. 154: 207-224.

Rovainen, C. M. (1979) Neurobiology of lampreys. Physiol. Rev. 59: 10071077

Rovainen, C. M. (1983) Neurophysiology. In The Biology of Lampreys, M. W. Hardisty and I. C. Potter, eds., Vol. 4a, pp. 1-136, Academic Press, London.

Rovainen, C. M., and K. L. Birnberger (1971) Identification and properties of motoneurons to fin muscle of the sea lamprey. J. Neurophysiol. 34: 974982.

Russell, D. F., and P. Wallén (1980) On the pattern generator for fictive swimming in the lamprey, Ichthyomyzon unicuspis. Acta Physiol. Scand. 108: 9 A.

Russell, D. F., and P. Wallén (1983) On the control of myotomal motoneurons during "fictive swimming" in the lamprey spinal cord in vitro. Acta Physiol. Scand. 117: 161-170.

Stewart, W. W. (1978) Functional connections between cells as revealed by dye-coupling with a highly fluorescent naphthalimide tracer. Cell 14: 741759.

Tang, D., and M. E. Selzer (1979) Projections of lamprey spinal neurons determined by the retrograde axon transport of horseradish peroxidase. J. Comp. Neurol. 188: 629-645.

Teräväinen, H. (1971) Anatomical and physiological studies on muscles of lamprey. J. Neurophysiol. 34: 954-973.

Teräväinen, H., and C. M. Rovainen (1971) Motoneurons to body muscle of the sea lamprey. J. Neurophysiol. 34: 990-998. 
Tretjakoff, D. (1909) Das Nervensystem von Ammocoetes. I. Das Rückenmark. Arch. Mikrosk. Anat. 73: 607-680.

van Dongen, P. A. M., T. Hökfelt, and S. Grilher (1983a) Neurons and fibers containing 5-hydroxytryptamine and peptides of the tachykinin-family in the lamprey spinal cord, as demonstrated by immunohistochemistry. J. Comp. Neurol., in press.

van Dongen, P. A. M., T. Hökfelt, S. Grillner, H. W. M. Steinbusch, A. A. J. Verhofstad, A. C. Cuello, and L. Terenius (1983b) 5-Hydroxytryptamine (5$\mathrm{HT}$ ), Substance $\mathrm{P}(\mathrm{SP})$ and Neuropeptide $\mathrm{Y}$ (NPY) immunoreactive neurons and axons in the spinal cord of lampreys. Acta Physiol. Scand. 120: 25A

van Dongen, P. A. M., T. Hökfelt, and S. Grillner (1983c) The relationship between 5 -hydroxytryptamine fibers and identified neurons in the lamprey spinal cord. A comination of transmitter histochemistry and intracellular staining with Lucifer yellow. J. Comp. Neurol., in press.

Wallén, P., and A. Lansner (1983) Are the motoneurones part of the central network generating the swimming rhythm in lamprey? Acta Physiol. Scand. 118: 6 A.

Wallén, P., and A. Lansner (1984) Do the motoneurones constitute a part of the spinal network generating the swirriming rhythm in the lamprey? J. Exp. Biol., in press.

Wallen, P., and T. L. Williams (1984) Fictive locomotion in the lamprey spinal cord in vitro compared with swimming in the intact and spinal animal. $J$. Physiol. (Lond.) 347: 225-239.

Wickelgren, W. O. (1977) Physiological and anatomical characteristics of reticulospinal neurones in lamprey. J. Physiol. (Lond.) 270: 89-114. 